\title{
INSATISFACCIÓN DE LA IMAGEN CORPORAL Y ESTADO NUTRICIONAL EN ESTUDIANTES UNIVERSITARIOS
}

\section{DISSATISFACTION WITH BODY IMAGE AND NUTRITIONAL STATUS IN UNIVERSITY STUDENTS}

\author{
Valeria Acuña Leiva \\ Programa de Magister en Psicoterapia Cognitiva Post-racionalista. Facultad de Psicología. \\ Universidad San Sebastián. Chile \\ ORCID: https://orcid.org/0000-0002-7415-4833

\section{Consuelo Niklitschek Tapia} \\ Programa de Magister en Psicoterapia Cognitiva Post-racionalista. Facultad de Psicología. \\ Universidad San Sebastián. Chile \\ ORCID: https://orcid.org/0000-0002-1153-1881
}

\section{Álvaro Quiñones Bergeret}

Facultad de Psicología, Universidad San Sebastián. Chile ORCID: https://orcid.org/0000-0002-6993-0324

\section{Carla Ugarte Pérez}

Facultad de Psicología, Universidad San Sebastián. Chile ORCID: https://orcid.org/0000-0002-8130-131X

Cómo referenciar este artículo/How to reference this article:

Acuña Leiva, V., Niklitschek Tapia, C., Quiñones Bergeret, A. y Ugarte Pérez, C. (2020). Insatisfacción De La Imagen Corporal y Estado Nutricional En Estudiantes Universitarios. Revista de Psicoterapia, 31(116), 279-294. https://doi.org/10.33898/rdp.v31i116.344 


\title{
Resumen
}

La imposición del modelo estético en las sociedades actuales, excesivamente delgado en las mujeres y musculoso para los hombres, lleva a que la representación corporal se vea muy influida por estos estándares externos, generando insatisfacción con el propio cuerpo, lo que puede desencadenar conductas alimentarias poco saludables. Los jóvenes universitarios son un grupo vulnerable debido a los cambios propios de la juventud. El objetivo del estudio fue describir el grado de insatisfacción con la imagen corporal, y su relación con el estado nutricional objetivo, el percibido y la concordancia en hombres y mujeres universitarios. Para ello se realizó un estudio transaccional de tipo cuantitativo con alcance descriptivo-correlacional. La muestra fue de 120 estudiantes universitarios (44 hombres y 76 mujeres) con edades comprendidas entre los 18 y 28 años. Se calculó el IMC, y se aplicaron los instrumentos Multidimensional Body Self Relations Questionnaire (MBSRQ), la sub-escala "Insatisfacción corporal” del Eating Disorders Inventory en su versión 2 (EDI-2), y un cuestionario sociodemográfico. El $17,5 \%(n=21)$ de la muestra sobreestimó su estado nutricional. De estos, un 90,5\% $(n=19)$ fueron mujeres. El IMC se asoció significativamente con la insatisfacción de la imagen corporal $(r=.45 ; p<.001)$. Los estudiantes que sobrestimación su estado nutricional presentaron mayores niveles de preocupación por el peso $(F=13,37$; $p<$ $.001)$ e insatisfacción con la imagen corporal $(F=7.24 ; p=.001)$ que los estudiantes que lo subestimaron o concordaron. La sobreestimación del estado nutricional se asocia a una mayor insatisfacción con la imagen corporal y una menor evaluación positiva de la apariencia. Ello podría desencadenar conductas alimentarias problemáticas, y trastornos de la conducta alimentaria, que permitiesen no autopercibirse tan distante del ideal estético cultural.

Palabras clave: Imagen corporal, Insatisfacción con la imagen corporal, Escalas de valoración, Índice de masa corporal.

\begin{abstract}
The imposition of the aesthetic model in today's societies, excessively thin in women and muscular for men, leads to the body representation being greatly influenced by these external standards, generating dissatisfaction. The objective this study was describe the degree of dissatisfaction with body image, and relationship with objective nutritional status, perception and concordance in university men and women. This was done transactional study of quantitative type with descriptive-correlational scope. The sample was 120 university students (44 men and 76 woman) whit ages between 18 and 28 years. BMI was calculated, and The Multidimensional Body Self Relations Questionnaire (MBSRQ), the "Body Dissatisfaction" sub-scale of the Eating Disorders Inventory in version 2 (EDI-2), and a sociodemographic questionnaire were applied. $17.5 \%(n=21)$ of the sample overestimated their nutritional status. Of these, $90.5 \%$ $(n=19)$ were women. BMI was directly and significantly associated with body image dissatisfaction $(r=.45 ; p<.001)$. The overestimation of nutritional status is significantly associated with concern about weight $(p<.05)$ and dissatisfaction with body image $(p=.001)$. Overestimation of nutritional status is associated with greater dissatisfaction with body image and less positive evaluation of appearance. This could trigger problematic eating behaviors, and eating disorders, which would make it possible to avoid making oneself so distant from the cultural aesthetic ideal.
\end{abstract}

Keywords: Body image, Body image dissatisfaction, Assessment scales, Body mass index. 


\section{Introducción}

La imagen corporal es la representación mental que cada persona construye en relación a su cuerpo y la vivencia en términos de sentimientos, conductas y actitudes que se tienen hacia el propio cuerpo (Baile, 2003; Raich, 2004; Rodríguez, Oudhof, González-Arratia y Unikel-Santocini, 2010). Se considera un constructo multidimensional complejo y dinámico (Baile, 2003). Además, la imagen corporal está conformada por tres componentes: perceptual, cognitivo-afectivo y conductual (Botella, Ribas y Benito, 2009; Kirszman y Salgueiro, 2015; Raich, 2004; Wertheim y Paxton, 2011). Para ser más específico, la distinción alude al modo en que el sujeto percibe, imagina, siente y actúa en relación a su propio cuerpo. En tal dirección, Slade (1994, p.502) señala "la imagen corporal es una representación mental amplia de la figura corporal, su forma y tamaño, la cual está influenciada por factores históricos, culturales, sociales, individuales y biológicos, que varían con el tiempo".

Desde una perspectiva evolutiva, la imagen corporal se va construyendo en la integración de cómo se siente y vive el cuerpo, a partir de cómo nos vemos y somos vistos desde niños (Beato-Fernández y Rodríguez-Cano, 2020; Pallan, Hiam, Duda y Adab, 2011). De hecho, se dispone de estudios que muestran que tanto niñas como niños entre los 5 y 7 años, reportan insatisfacción, preocupación y percepción inexacta de la imagen corporal (Pallan et al., 2011) la que se ha observado incluso desde los tres años (Tremblay, Lovsin, Zecevic y Larivière, 2011).

Por otra parte, la imagen corporal influye en el procesamiento de la información. A saber, la manera en cómo percibimos el mundo está influenciada por la forma en que sentimos y pensamos acerca de nuestro propio cuerpo, pudiendo sentirnos satisfechos o insatisfechos con esa imagen (Baile, 2003). Más aún, según Pruzinsky y Cash (1990), Cash (2004) y Cash y Smolak (2011), existirían varias imágenes corporales interrelacionadas: una imagen perceptual, una imagen cognitiva y una imagen emocional. Es de destacar que la imagen emocional incluye los sentimientos sobre el grado de satisfacción con nuestra figura y con las experiencias que nos proporciona nuestro cuerpo.

Según el metanálisis de Cash y Deagle (1997), la imagen corporal perceptiva representa la precisión con la que una persona puede juzgar las dimensiones físicas de su propio cuerpo. La autopercepción se ha catalogado como correcta (coincide con los datos objetivos de IMC o volumen corporal), subestimada (se autopercibe con un menor IMC del que tiene, o un menor volumen corporal) y sobrestimada (se autopercibe con un mayor IMC del que tiene, o mayor volumen corporal); y el tipo de valoración podría generar satisfacción o insatisfacción corporal. Existen autores que consideran que la distorsión perceptiva es la alteración de la percepción que se manifiesta por una incapacidad para estimar con exactitud el tamaño corporal (Contreras, Gil-Madrona, Garcia-Lopez, Fernández-Bustos y Pastor-Vicedo, 2012).

En relación a la insatisfacción con la imagen corporal, algunos estudios longitudinales han informado una mayor insatisfacción con la imagen corporal en 
adultos jóvenes que en adolescentes (Bearman, Presnell, Martinez y Stice, 2006; Bucchianeri, Arikian, Hannan, Eisenberg y Neumark-Sztainer, 2013).

Desde una perspectiva de investigación en estudiantes universitarios (adulto emergente) (Arnett, 2000; Barrera-Herrera y Vinet, 2017), mencionaremos algunos estudios que nos parecen pertinentes. Un estudio realizado en España por Míguez, De la Montaña, Gonzálezy González (2011) en una muestra 145 universitarios, cuyo objetivo fue detectar posibles alteraciones de la conducta alimentaria mediante la autopercepción de su imagen corporal, encontró que más de la mitad de las mujeres y de los hombres que comprendio la muestra (55\% de las mujeres, y el 63\% de los hombres) se percibió de manera distinta respecto a los valores del índice de masa corporal (IMC), observándose que los hombres subestimaron su peso y las mujeres subestimaron y sobreestimaron su peso. Además, este estudio encontró que las mujeres que estaban más insatisfechas con su imagen corporal presentaron sobrepeso o bajo peso y las más preocupadas por adelgazar se encontraron en el límite superior del normopeso. En relación a los hombres, los que presentaron sobrepeso y obesidad fueron los más insatisfechos y los más obsesionados por adelgazar. En otro estudio desarrollado en el mismo país y dirigido por Soto et al. (2015), cuyo objetivo consistió en describirla percepción de la imagen corporal de 1162 estudiantes universitarios, se encontró que el 55,6\% tuvo una percepción de imagen corporal que no se correspondía con los datos objetivos (IMC). El 9.7\% de los hombres y el $58.1 \%$ de las mujeres sobreestimaron su IMC. Los autores también encontraron que un porcentaje importante de estudiantes presentaron alteraciones en la percepción de su imagen corporal, siendo estas más frecuentes en mujeres. Específicamente, encontraron que las mujeres tenían una mayor tendencia a sobreestimar su IMC en comparación con los hombres, cuya tendencia fue a subestimarlo.

En cuanto a las investigaciones realizadas en Chile, merece especial mención el estudio realizado por Mujica et al. (2009), con una muestra de 1007 sujetos, cuyo objetivo fue determinar la concordancia entre el estado nutricional y la autopercepción del estado de peso en adultos entre 18 y 74 años. Se obtuvo como resultado que el $73 \%$ de los sujetos tenían sobrepeso u obesidad y el $44 \%$ subestimó su estado nutricional. El 60\% de los sujetos con un IMC entre 25 y $26 \mathrm{~kg} / \mathrm{m} 2$, se percibieron a sí mismos como normales. Se observó una mayor subestimación en sujetos con obesidad, en individuos con sobrepeso, en hombres y en personas de 45 a 59 años. Este último grupo presentó un $70 \%$ más de riesgo de subestimar su estado nutricional al ser comparado con el grupo etario de 18 a 29 años. Los autores concluyeron que cerca de la mitad de la población estudiada tuvo una autopercepción errónea del peso, principalmente debido a la subestimación.

Más recientemente en nuestro país, Cruzat-Mandich et al. (2016, 2017) han investigado la imagen corporal y su insatisfacción en distintas muestras de jóvenes. En una muestra de 1438 adolescentes y jóvenes de los cuales el 68,3\% tenía entre 18 y 25 años, las investigadoras encontraron que el 70\% de las adolescentes y jóvenes de sexo femenino querían estar más delgadas, mostraban mayor preocu- 
pación por el peso y presentaban mayores niveles de insatisfacción de la imagen corporal que sus pares masculinos (Cruzat et al., 2016). En otro estudio Cruzat et al. (2017a) en 376 jóvenes de 18 a 25 años con normopeso y malnutrición por exceso, encontraron que los jóvenes con sobrepeso/obesidad presentaron mayor insatisfacción con la imagen corporal $(p<0.001)$, peor evaluación de su condición física $(p<0.05)$ y mayor preocupación por el peso $(p<0.001)$ que los jóvenes en el rango de normopeso respecto al IMC (Cruzat et al., 2017a).

En un estudio intercultural entre estudiantes universitarios chilenos y panameños, Durán et al. (2013) buscaron determinar la asociación entre el estado nutricional y la percepción de la imagen corporal en una muestra de 792 estudiantes. Los resultados señalaron que el IMC percibido fue sobreestimado en el $60 \%$ de los chilenos. En cambio, en la muestra panameña la sobreestimación es inferior al 50\%. En ambos países, solo un 20\% de los sujetos se percibió como realmente son. En la muestra chilena, específicamente se encontró que existe concordancia entre el IMC y la imagen corporal en la mitad de los hombres chilenos y un tercio de las mujeres chilenas. Sin embargo, se observa una mejor concordancia en estudiantes con sobrepeso (69\% en hombres; $90.5 \%$ en mujeres). En cambio, en los panameños, se observa una concordancia del $40 \%$ en hombres y del $19.9 \%$ en mujeres. En síntesis, en ambas muestras universitarias, los estudiantes en el rango de normopeso presentan una tendencia a la sobreestimación. Sin embargo, los estudiantes con obesidad tienden a subestimar su peso.

Debido a las características de prevalencia de desórdenes alimentarios y obesidad en Chile, destacamos los siguientes estudios, a modo de contextualizar con mayor precisión la información.

Por una parte, los estudios en general en muestras de personas jóvenes y adultas, la insatisfacción con la imagen corporal se encuentra entre los factores más relevantes que condicionan la ingesta de alimentos y el peso corporal (Cáceres, 2005; Fehrman-Rosas et al., 2016; Gismero González, 2020; Ramos, Rivera y Moreno, 2010; Ramos, Rivera, Pérez, Lara y Moreno, 2016; Williamson et al., 2000). De igual manera, puede conducir a prácticas alimentarias de riesgo (Treasure y Schmidt, 2013) y puede ser un factor predisponente y precipitante para el desarrollo de una distorsión de la imagen corporal, criterio diagnóstico y psicopatológico de trastornos de la conducta alimentaria y la ingesta de alimentos como la anorexia y la bulimia (5. ${ }^{\mathrm{a}}$ Ed.; DSM-V; American Psychiatric Association [APA], 2013; Fairburn, Cooper, y Shafran, 2003; Mölbert et al., 2017). En este sentido, la detección precoz de la insatisfacción de la imagen corporal y sus componentes, puede tener también un papel relevante en la prevención de los trastornos de la conducta alimentaria.

Por otra parte, diversos estudios también muestran que la insatisfacción con la imagen corporal es mayor en personas con malnutrición por exceso (Cruzat-Mandich et al., 2017; Trejo, Castro, Facio, Mollinedo y Valdez, 2010; Streeter, Milhausen y Buchholz, 2012). Tanto a nivel mundial como local, el sobrepeso y la obesidad son un problema de salud de relevancia mundial (OMS, 2018; MINSAL, 2017a). 
Específicamente, Chile hasta el año 2016 poseía el segundo lugar de malnutrición por exceso a nivel mundial, con un 34,4\% de obesidad en población adulta (FAO, 2017). Es más, la última Encuesta Nacional de Salud mostró que el sobrepeso y la obesidad siguen en aumento a nivel nacional (MINSAL, 2017a), ello pese a la implementación de la Ley 20.606 (MINSAL, 2017b) y diversos programas como "elije vivir sano" que han intentado mitigar esta situación. Además en nuestro país, el número de cirugías bariátricas tanto en el sector privado como en el sector público ha ido aumentando año tras año (Csendes, 2015; Guzmán et al., 2013). No obstante, todas estas intervenciones no han tenido el impacto esperado en relación a frenar la prevalencia de sobrepeso y obesidad, que sigue en aumento (Ugarte, Quiñones y Vicente, 2019).

El presente estudio tiene como propósito abordar la relación entre la imagen corporal y la insatisfacción con la imagen en relación al estado nutricional objetivo según IMC, el estado nutricional percibido y la concordancia entre ambos.

\section{Objetivo}

El objetivo de este estudio fue describir el grado de insatisfacción con la imagen corporal entre hombres y mujeres, y su relación con el estado nutricional objetivo, el percibido y la concordancia entre estos, en estudiantes de Psicología de la Universidad San Sebastián.

\section{Método}

Se realizó un estudio cuantitativo de alcance descriptivo-correlacional, mediante diseño no experimental sobre la base de una encuesta transversal. La muestra estuvo conformada por 120 estudiantes (44 hombres y 76 mujeres) de la carrera de Psicología de la Universidad San Sebastián, sede Concepción. Se incluyeron estudiantes de los cursos comprendidos entre $1^{\circ}$ y $5^{\circ}$ año, seleccionados en base a un muestreo no probabilístico por accesibilidad a la muestra.

Los criterios de inclusión fueron ser estudiante universitario mayor de 18 años y sin discapacidad visual. Y los de exclusión, presentar limitaciones senso-perceptivas (particularmente limitación visual) que impidiesen el desempeño en las pruebas.

Los participantes respondieron en forma auto-administrada un cuestionario que incluía datos sociodemográficos construido especialmente para esta investigación. Además, de las escalas "Multidimensional Body Self Relations Questionnaire (MBSRQ)", y la subescala "Insatisfacción corporal” del "Eating Disorders Inventory en su versión 2 (EDI-2)”.

\section{Variables de resultado}

Estado nutricional objetivo: se determinó por medio del cálculo de IMC. Este índice se calculó dividiendo el peso por la talla al cuadrado (IMC= peso en $\mathrm{Kg} / \mathrm{talla}^{2}$ en metros). De esta forma, el estado nutricional se clasificó según las normas de la Organización Mundial de la Salud como: bajo peso $<18.5 \mathrm{Kg} / \mathrm{m}^{2}$, normal 18.5 a 24.9 
$\mathrm{Kg} / \mathrm{m}^{2}$, sobrepeso 25 a $29.9 \mathrm{Kg} / \mathrm{m}^{2}$ y obesidad IMC $\geq 30 \mathrm{Kg} / \mathrm{m}^{2}$. Para el cálculo del IMC, se utilizó una balanza marca Seca 762, la cual es una báscula mecánica con fina graduación, con una capacidad máxima de $150 \mathrm{~kg}$ y un margen de error de \pm 50 gramos. La talla se midió con un estadímetro móvil marca Seca 217, mide hasta una talla de $205 \mathrm{~cm}$, con un margen de error de $\pm 1 \mathrm{~mm}$.

Estado nutricional percibido: Fue evaluado a partir de la autopercepción del estado nutricional de los estudiantes. Para consignar su autopercepción nutricional, en el dossier de instrumentos se les realizó la pregunta “¿Cómo se considera usted en relación a su estado nutricional?” cuyas alternativas de respuesta posibles eran "con bajo peso", “con peso normal”, “con sobrepeso", “con obesidad”.

Concordancia entre estado nutricional objetivo y percibido: En relación al grado de concordancia entre el estado nutricional objetivo y el subjetivo (auto-percepción) se distinguieron tres posibilidades según la propia clasificación del sujeto. Primero: se consideró subestimación de peso cuando se clasificó en una categoría de estado nutricional inferior a la correspondiente según el criterio clínico (IMC). Segundo: se estableció como sobrestimación del propio peso cuando se clasificó en una categoría mayor a la correspondiente. Tercero: se estableció como concordancia cuando se clasificó en la misma categoría de estado nutricional según criterio clínico.

\section{Instrumentos psicométricos}

Multidimensional Body Self Relations Questionnaire(MBSRQ): Cuestionario que evalúa aspectos actitudinales respecto a la imagen corporal y actitudes respecto de la propia apariencia física. Fue creado por Brown, Cash y Mikulka (1990), para evalúar los componentes de: satisfacción con el aspecto de uno mismo, preocupación por la imagen corporal, autopercepción de la forma física, evaluación del propio estado de salud/enfermedad, intención de seguir un estilo de vida saludable, preocupación por el peso, auto clasificación respecto al peso y satisfacción con varias zonas del cuerpo. Es un cuestionario autoaplicado que consta de 69 ítems que aluden a distintas actitudes hacia la imagen corporal, se responde en formato Likert de cinco puntos donde 1 es "totalmente en desacuerdo" a 5 "totalmente de acuerdo", según el grado en que se sienta identificado con el ítem. Su versión en español fue validada por Botella, Rivas y Benito (2009), y en Chile fue validada por Cruzat et al. (2017b). La versión al castellano (Botella et al., 2009) obtuvo índices de consistencia interna superiores a $\alpha=0.7$ para todas las subescalas. El análisis factorial de la validación chilena Cruzat el at. (2017b), informó una estructura de siete factores. Factor I (Orientación y evaluación de la actividad física), Factor II (Evaluación de la apariencia), Factor III (Preocupación por el peso), Factor IV (Orientación hacia la apariencia), Factor V (Orientación hacia la salud), Factor VI (Evaluación de enfermedad) y Factor VII (Orientación hacia la enfermedad). Y la consistencia interna de los factores fue de $\alpha=0.92$ para orientación y evaluación de la actividad física (Factor I), $\alpha=0.91$ para evaluación de la apariencia (Factor II), $\alpha=0.70$ para preocupación por el peso (Factor III), $\alpha=0.84$ para orientación 
hacia la apariencia (Factor IV), $\alpha=0.74$ para orientación hacia la salud (Factor $\mathrm{V}$ ), $\alpha=0.70$ para evaluación de enfermedad (Factor VI), y $\alpha=0.70$ para orientación hacia la enfermedad (Factor VII).

Sub-escala Insatisfacción corporal del Eating Disorders Inventory en su versión 2 (EDI-2): La escala EDI-2 fue creada por David Garner en 1983. Es una escala que mide síntomas psicológicos asociados comúnmente a la anorexia nerviosa, la bulimia nerviosa y otros trastornos alimentarios. En población Chilena fue adaptada por Urzúa, Castro, Lillo y Leal (2009). La escala actual de EDI-2 evalúa 11 subescalas a través de 91 ítems. Para la presente investigación solo se utilizó la subescala "insatisfacción corporal" compuesta por 9 ítems, con un formato de respuestas en una escala tipo Likert de seis puntos: "siempre", "casi siempre”, "frecuentemente", "en ocasiones", "rara vez” o "nunca”. Esta sub-escala ha mostrado buenos índices de fiabilidad en población chilena $(\alpha=0.77)$.

Cuestionario sociodemográfico. Se construyó un cuestionario ad hoc para la presente investigación. Este cuestionario consignó datos como sexo, edad, año de carrera y antecedentes mórbidos.

\section{Análisis estadísticos}

Las variables fueron descritas según normalidad de acuerdo al test de Shapiro Wilk. Se utilizó la prueba $t$ de Student para estudiar la relación entre el sexo y la imagen corporal e insatisfacción con la imagen corporal. Para evaluar si los estudiantes presentaban diferencias en la insatisfacción con la imagen corporal según el grado de concordancia con su estado nutricional objetivo (concuerda, subestima, sobreestima) se utilizó ANOVA de un factor. Para evaluar la asociación entre las variables numéricas se utilizó el coeficiente de Pearson. Por último, para analizar la relación entre el género y el grado de concordancia se utilizó la prueba Chi Cuadrado.

Consideraciones éticas: Todos los participantes firmaron un Consentimiento Informado.Además, para esta investigación se realizó una revisión de los principales protocolos de principios éticos para la investigación en humanos, Declaración de Helsinkin en español 2013, Código de Núremberg y Normas de buenas prácticas clínicas (BPC), tanto en la recolección y codificación de los datos para el resguardo de la información de acuerdo a los requerimientos de ética correspondientes.

\section{Resultados}

De la muestra un 37.2\% $(n=44)$ eran hombres y un 62.8\% $(n=76)$ mujeres. El promedio de edad fue de 21.4 años $(D E=2)$, con un mínimo de 18 años y un máximo de 28 años.

El IMC promedio fue de $24.6 \mathrm{~kg} / \mathrm{m} 2(D E=4.3)$, con un mínimo de $17.4 \mathrm{~kg} /$ m2 y un máximo de $44.9 \mathrm{~kg} / \mathrm{m} 2$. En cuanto al sexo, se encontró que el promedio de IMC en hombres fue un $25.1 \mathrm{~kg} / \mathrm{m} 2$ y en mujeres $24.4 \mathrm{~kg} / \mathrm{m} 2$.

En cuanto al estado nutricional objetivo un $60.8 \%$ de la muestra se clasificó 
según su IMC en la categoría normopeso, un $27.5 \%$ en sobrepeso y un $11.7 \%$ en obesidad. Respecto al estado nutricional percibido, el $51.7 \%$ de los participantes se autoclasificó en una categoría de "con normopeso", un 41.7\% en "con sobrepeso" y un $6.6 \%$ en la categoría "con obesidad". El detalle de la distribución por sexo se muestra en la Tabla 1.

En otro orden de cosas, el análisis de fiabilidad de los instrumentos para este estudio, indican un alfa de cronbach de $\alpha=0.83$ para la subescala de insatifacción de imagen corporal del EDI-2. El alfa de Cronbach para los factores de la escala Multidimensional Body Self Relations Questionnaire, fue de $\alpha=0.91$ para el Factor I (Orientación y evaluación de la actividad física) y II (Evaluación de la apariencia), $\alpha=0.66$ para el Factor III (Preocupación por el peso), $\alpha=0.82$ para el Factor IV (Orientación hacia la apariencia), $\alpha=0.74$ para el Factor V (Orientación hacia la salud), $\alpha=0.71$ para el Factor VI (Evaluación de enfermedad), y $\alpha=0.51$ para el Factor VII (Orientación hacia la enfermedad).

Tabla 1. Distribución por sexo en estado nutricional objetivo y percibido.

\begin{tabular}{lcccccc}
\hline Sexo & \multicolumn{3}{c}{ Estado nutricional objetivo } & \multicolumn{3}{c}{ Estado nutricional autopercibido } \\
\cline { 2 - 7 } & $\begin{array}{c}\text { Normopeso } \\
\mathbf{n}(\%)\end{array}$ & $\begin{array}{c}\text { Sobrepeso } \\
\mathbf{n}(\%)\end{array}$ & $\begin{array}{c}\text { Obesidad } \\
\mathbf{n}(\%)\end{array}$ & $\begin{array}{c}\text { Normopeso } \\
\mathbf{n}(\%)\end{array}$ & $\begin{array}{c}\text { Sobrepeso } \\
\mathbf{n}(\%)\end{array}$ & $\begin{array}{c}\text { Obesidad } \\
\mathbf{n}(\%)\end{array}$ \\
\hline Hombre & $24(54.5 \%)$ & $14(31.8 \%)$ & $6(13.6 \%)$ & $27(61.4 \%)$ & $16(36.4 \%)$ & $1(2.3 \%)$ \\
\hline Mujer & $49(64.5 \%)$ & $19(25 \%)$ & $8(10.5 \%)$ & $35(46.1 \%)$ & $34(44.7 \%)$ & $7(9.2 \%)$ \\
\hline
\end{tabular}

Los análisis no muestran diferencias significativas entre el género y el estado nutricional objetivo $\left(X^{2}=1.15 ; p=0.5\right)$ y autopercibido $\left(X^{2}=3.74 ; p=0.15\right)$ cuando se analizan de forma diferencial.

Ahora bien, en relación al grado de concordancia entre el estado nutricional objetivo y autopercibido se encontró que el $69.2 \%(n=83)$ de los participantes se clasificó correctamente en su estado nutricional objetivo. Mientras que el 17,5\% ( $n$ $=21)$ sobreestimó su estado nutricional y el $13.3 \%(n=16)$ lo subestimó. De los 16 estudiantes que subestimaron su peso, el 62.5\% $(n=10)$ correspondió al sexo masculino. Mientras que de los 21 estudiantes que sobrestimaron su peso, el $90.5 \%$ $(n=19)$ fueron de sexo femenino. Estas diferencias entre el grado de concordancia y el sexo fueron significativas $\left(X^{2}=11.39 ; p=0.003\right)$ y se presentan en la tabla 2 .

Tabla 2. Grado de concordancia entre IMC objetivo y autopercibido y sexo.

\begin{tabular}{cccc}
\hline Sexo & \multicolumn{3}{c}{ Grado de concordancia } \\
\cline { 2 - 4 } & $\begin{array}{c}\text { Subestima } \\
\mathbf{n}(\%)\end{array}$ & $\begin{array}{c}\text { Concuerda } \\
\mathbf{n}(\%)\end{array}$ & $\begin{array}{c}\text { Sobrestima } \\
\mathbf{n}(\%)\end{array}$ \\
\hline Hombre & $10(62.5 \%)$ & $32(38.6 \%)$ & $2(9.5 \%)$ \\
\hline Mujer & $6(37.5 \%)$ & $51(61.4 \%)$ & $19(90.5 \%)$ \\
\hline Total & $16(100 \%)$ & $83(100 \%)$ & $21(100 \%)$ \\
\hline
\end{tabular}


Respecto a la relación entre el sexo, imagen corporal e insatisfacción con la imagen corporal, se observaron diferencias significativas entre hombres y mujeres solo en los factores del MBSRQ “orientación y evaluación de la actividad física”, factor en que los hombres presentaron una media significativamente mayor ( $M=$ 56.2; $D E=17.3$ versus $\mathrm{M}=45.2 ; D E=12.7 ; t=4 ; p<0.001$ ), y "Orientación hacia la apariencia", factor en el que la media de las mujeres es significativamente mayor $(p<0.05)$. El tamaño del efecto fue de 0.72 (gran efecto) para el Factor I, y de 0.39 (efecto pequeño) para el Factor IV (Cohen, 1988) (veáse tabla 3).

Tabla 3. Relación entre sexo, imagen corporal e insatisfacción con imagen corporal.

\begin{tabular}{|c|c|c|c|c|}
\hline Variable & $\begin{array}{l}\text { Hombre } \\
M(D E)\end{array}$ & $\begin{array}{l}\text { Mujer } \\
M(D E)\end{array}$ & $t$ & Cohen's d \\
\hline $\begin{array}{l}\text { Factor I (Orientación y evaluación de la } \\
\text { actividad física) }\end{array}$ & $56.2(17.3)$ & $45.2(12.7)$ & $4^{\star \star \star}$ & 0.72 \\
\hline Factor II (Evaluación de la apariencia) & $44.7(15.8)$ & $42.8(12.4)$ & 0.73 & - \\
\hline Factor III (Preocupación por el peso) & $11.16(4.9)$ & $12.3(4.3)$ & -1.33 & - \\
\hline $\begin{array}{l}\text { Factor IV (Orientación hacia la aparien- } \\
\text { cia) }\end{array}$ & $45.9(12.8)$ & $50.0(8)$ & $-2.16^{\star}$ & 0.39 \\
\hline Factor V (Orientación hacia la salud) & $24.1(8.6)$ & $24.0(5.4)$ & 0.05 & - \\
\hline Factor VI (Evaluación de enfermedad) & $11.1(6)$ & $12.6(4)$ & -1.6 & - \\
\hline $\begin{array}{l}\text { Factor VII (Orientación hacia la enfer- } \\
\text { medad) }\end{array}$ & $18.6(6.3)$ & $17.3(4)$ & 1.4 & - \\
\hline $\begin{array}{c}\text { Sub-escala EDI-2 } \\
\text { Insatisfacción con imagen corporal }\end{array}$ & $21.4(12.2)$ & $12.2(10.2)$ & -1.24 & - \\
\hline
\end{tabular}

Nota. ${ }^{*} p<0.05,{ }^{* *} p<0.01,{ }^{* *} p<0.001$.

A continuación, el análisis de correlaciones mostró una asociación directa y significativa entre el IMC de los estudiantes y la insatisfacción de la imagen corporal, evaluada a través de la sub-escala del EDI-2 ( $r=0.45 ; p<0.001)$. Es decir, a mayor IMC mayores niveles de instafisfacción con la imagen corporal.

En cuanto a la asociación del IMC con las subescalas del MBSRQ, las correlaciones mostraron asociaciones significativas solo en los factores II y III. Se observó una asociación inversa entre el factor II (evaluación de la apariencia) y el IMC ( $r=-0.3$; $p=0.001)$, y una asociación directa entre el IMC y el factor III (preocupación por el peso) $(r=0.38 ; p<0.001)$. Por lo que a mayor IMC se observa una menor evaluación positiva de la apariencia y mayor preocupación por el peso.

En último lugar, se evaluaron las diferencias en imagen corporal e insatisfacción con la imagen corporal, según grado de concordancia con estado nutricional. En este caso se encontraron diferencias significativas en dos factores del MBRSQ, Factor III (preocupación por el peso) $(F=13.37$; $p<0.001$ ) y Factor IV (orientación hacia la apariencia) ( $F=9.22 ; p<0.001)$. En el factor VI (evaluación de enfermedad) las diferencias estuvieron cercanas a la significancia $(F=2.94 ; p=0.057)$. Además, se encontraron diferencias en la insatisfacción de la imagen corporal $(F=7.24 ; p$ 
$<0.001$ ) (véase tabla 4).

Tabla 4. Relación entre imagen corporal e insatisfacción con el grado de concordancia entre IMC objetivo y autopercibido.

\begin{tabular}{|c|c|c|c|c|}
\hline Variable & $\begin{array}{l}\text { Subestima } \\
M(D E)\end{array}$ & $\begin{array}{l}\text { Concuerda } \\
M(D E)\end{array}$ & $\begin{array}{l}\text { Sobreestima } \\
M(D E)\end{array}$ & $F$ \\
\hline $\begin{array}{l}\text { Factor I (Orientación y evaluación de la acti- } \\
\text { vidad física) }\end{array}$ & $50.7(12.8)$ & $48.5(12.8)$ & $46.8(13.8)$ & 0.42 \\
\hline Factor II (Evaluación de la apariencia) & $41(9.45)$ & 43.9(11.5) & 39.7(13.1) & 1.32 \\
\hline Factor III (Preocupación por el peso) & $12(2.4)$ & $10.7(2.9)$ & $15.2(5.5)$ & $13.37^{\star \star \star}$ \\
\hline Factor IV (Orientación hacia la apariencia) & $47.5(8)$ & $46.4(7.99)$ & $54.3(5.7)$ & $9.22^{\star \star \star}$ \\
\hline Factor V (Orientación hacia la salud) & $23.8(6)$ & $23.5(5.2)$ & $24(4.4)$ & 0.068 \\
\hline Factor VI (Evaluación de enfermedad) & $11.7(6)$ & $11.4(4.1)$ & $14.1(2.9)$ & 2.94 \\
\hline Factor VII (Orientación hacia la enfermedad) & $17.2(3.5)$ & $17.6(3.9)$ & $17.2(3.8)$ & 0.11 \\
\hline $\begin{array}{l}\text { Insatisfacción con imagen corporal } \\
\text { (EDI-2) }\end{array}$ & $22.7(8)$ & $20.8(9.4)$ & $29.4(9)$ & $7.24^{\star \star}$ \\
\hline
\end{tabular}

Nota. ${ }^{\star} p<0.05,{ }^{\star \star} p<0.01,{ }^{\star \star \star} p<0.001$.

Para analizar entre qué grupos específicamente se presentan las diferencias significativas, se realizó un análisis post hoc mediante la prueba Bonferroni. Los resultados mostraron que los estudiantes que sobrestimaron su estado nutricional presentaron mayores niveles de preocupación por el peso (diferencia de medias = 3.17; $D E=1.22 ; p<0.05$ ) y mayor orientación hacia la apariencia (diferencia de medias $=6.8 ; D E=2.5 ; p<0.05$ ) que los que subestimaron su peso. También, los estudiantes que sobreestimaron su peso presentaron mayores niveles de preocupación por el peso (diferencia de medias $=4.4 ; D E=0.87 ; p<0.001$ ) y mayor orientación hacia la apariencia que los que acertaron en su autopercepción (diferencia de medias $=7.97 ; D E=1.8 ; p<0.001$ ). Finalmente, la insatisfacción con la imagen corporal fue significativamente mayor solo entre las personas que sobreestimaron su estado nutricional versus los que se autodefinieron correctamente (Diferencia de medias = 8.6; $D E=2.2 ; p=0.001$ ).

\section{Discusión}

La presente investigación tuvo por objeto describir el grado de insatisfacción con la imagen corporal en hombres y mujeres y su relación con el estado nutricional objetivo, el percibido y la concordancia entre ambos en estudiantes universitarios.

Los resultados encontrados se discuten a continuación:

En primer lugar, se debe destacar que un 38.7\% de la muestra presentó un IMC correspondiente a sobrepeso u obesidad. Este porcentaje de estudiantes con malnutrición por exceso confirma que las prevalencias del sobrepeso y la obesidad en estudiantes universitarios son elevadas (Durán, Valdés, Godoy y Herrera, 2014).

En segundo lugar, en cuanto a la autopercepción del estado nutricional, se encontró que casi un 70\% de los participantes se autopercibió correctamente. Este 
resultado encontrado en nuestra investigación, no concuerda con los porcentajes reportados en los estudios revisados (Durán et al., 2013; Mujica et al., 2008), los que reportan una autopercepción no concordante en población universitaria. específicamente el estudio de Durán et al. (2013) encontró mayores niveles de sobreestimación. Este hallazgo puede explicarse en función de la forma en que se consignó dicha información. Ello debido a que no se utilizó imágenes de modelos anatómicos para dicho fin, sino más bien se consignó a través de una pregunta y respuesta escrita.

Ahora bien, el 25.7\% de la muestra que presentó un IMC menor a 25 sobreestimó su estado nutricional. Mientras que un $21.2 \%$ y un $64.3 \%$ de los estudiantes con IMC de sobrepeso u obesidad, lo subestimaron. Este resultado, sí concuerda con las investigaciones realizadas por Durán et al. (2013) respecto a la tendencia de las personas con normopeso a sobreestimar su estado nutricional, y la tendencia a la subestimación de los grupos con obesidad y sobrepeso (Mujica et al., 2008).

En tercer lugar, en relación a la asociación entre imagen corporal y sexo, nuestros resultados muestran que los hombres presentaron mayores puntuaciones en el área de la evaluación de la actividad física y las mujeres mostrarón una mayor orientación hacia la apariencia. Esto fue coherente con los estudios que señalan que los hombres están más preocupados por el desarrollo muscular y las mujeres por la delgadez (Cruzat-Mandich et al., 2016; Tiggemann, Martins y Churchett, 2008).

En cuanto a la satisfacción con la imagen corporal, se observó que a mayor IMC mayor insatisfacción con la imagen corporal, mayor preocupación por el peso y una peor evaluación de la apariencia física. Este resultado es concordante con las investigaciones en el área, que evidencian que las personas con un mayor IMC presentan mayores niveles de insatisfacción en estas tres áreas (Cruzat-Mandich et al., 2017; Trejo et al., 2010; Streeter et al., 2012).

También los resultados obtenidos muestran que, los estudiantes que sobreestimaron su estado nutricional presentaron mayores niveles de preocupación por el peso, mayor orientación y preocupación por la apariencia y mayor insatisfacción con la imagen corporal que los estudiantes que se autopercibieron correctamente o subestimaron su estado nutricional. Este resultado es coherente y esperable de acuerdo a la literatura (Cruzat-Mandich et al., 2017; Trejo et al., 2010; Streeter et al., 2012). El estudiante al autopercibir su estado nutricional de forma sobreestimada, visualiza que su volumen corporal se distancia en mayor medida del ideal estético cultural, lo que incrementa la insatisfacción. La insatisfacción por tanto, en estos casos es independiente del estado nutricional real (IMC). Ello podría generar el deseo una silueta más delgada a la percibida (Díaz et al., 2019), y en consencuencia desencadenar conductas alimentarias no saludables y eventualmente un trastorno de la conducta alimentaria (Cruzat-Mandich et al., 2017; Díaz et al., 2019).

Para concluir, nuestros resultados en población universitaria, indican que la insatisfacción corporal está más relacionada con la percepción subjetiva del peso que con el peso objetivo. Nuestra interpretación es que la imagen corporal 
percibida es más relevante en función de la imposición del modelo estético, excesivamente delgado en las mujeres y musculoso para los hombres, que es propio de las sociedades occidentales (Craig, Swinburn, Matenga-Smith, T., Matangi, y Vaughn, 1996; Craig, Halavatau, Comino y Caterson, 1999). Esto lleva a que la configuración y representación corporal se vea muy influida por estos estándares culturales, generando frustración e insatisfacción con la imagen corporal cuando no se ha alcanzado dicho estándar. Esto es un aspecto a tomar en consideración en salud pública, puesto que esta insatisfacción con la imagen corporal puede ser un factor de riesgo para el desarrollo de trastornos de la conducta alimentaria alimentaria (Cruzat-Mandich et al., 2017; Díaz et al., 2019).

\section{Limitaciones del estudio}

Las limitaciones de este estudio fueron el tipo de muestreo, el tamaño muestral pequeño y una muestra predominante de mujeres.

\section{Líneas futuras de investigación}

Se sugiere realizar estudios aleatorios con muestras más grandes, de distintas carreras, que permita generalizar los resultados al grupo de edad. Asimismo, evaluar la presencia y relación entre los constructos evaluados y los síntomas de trastornos alimentarios en este tipo de población.

\section{Referencias Bibliográficas}

American Psychiatric Association. (2013). Feeding and Eating Disorders. In Diagnostic and Statistical Manual of Mental Disorders (DSM-5) (5 ${ }^{\mathrm{a}}$ ed.). Washington, DC: APA.

Arnett, J. J. (2000). Emerging adulthood: A theory of development from the late teens through the twenties. American Psychologist, 55(5), 469-480. https://doi.org/10.1037/0003-066X.55.5.469

Baile, J. (2003). ¿Qué es la imagen corporal? Revista de Humanidades Cuadernos del Marqués de San Adrian, 2, 53-72. Recuperado de: https://dialnet.unirioja.es/ejemplar/135873

Barrera, A. y Vinet, E. (2017). Adultez Emergente y características culturales de la etapa en universitarios chilenos. Terapia psicológica, 35(1), 47-56. https://dx.doi.org/10.4067/S0718-48082017000100005

Bearman, S. K., Presnell, K., Martinez, E. y Stice E. (2006). The skinny on body dissatisfaction: A longitudinal study of adolescent girls and boys. Journal of Youth and Adolescence, 35, 229-241. https://doi.org/10.1007/ s10964-005-9010-9

Beato-Fernández, L. y Rodríguez-Cano, T. (2020). Identidad y Proceso Emocional de Cambio en los Trastornos del Comportamiento Alimentario [Identity and Emotional Process of Change in Eating Disorders]. Revista de Psicoterapia, 31(115), 17-31. https://doi.org/10.33898/rdp.v31i115.333

Botella, L., Ribas, E. y Benito, J. (2009). Evaluación psicométrica de la imagen corporal: validación de la versión española del Multidimensional Body Self Relations Questionnaire (MBSRQ). Revista Argentina de Clinica Psicológica, 28(3), 253-264. Recuperado de: https://www.redalyc.org/pdf/2819/281921775006.pdf

Bucchianeri, M. M., Arikian, A. J., Hannan, P. J., Eisenberg, M. E. y Neumark-Sztainer, D. (2013). Body dissatisfaction from adolescence to young adulthood: findings from a 10-year longitudinal study. Body image, 10(1), 1-7. https://doi.org/10.1016/j.bodyim.2012.09.001

Cáceres, J. J. (2005). La incidencia de la preocupación por la imagen corporal e las elecciones alimentarias de los jóvenes. Zainak Cuadernos de Antropología-Etnografía, 27, 165-77. Recuperado de: https://core.ac.uk/ download/pdf/11499086.pdf

Cohen, J. (1988). Statistical Power Analysis for the Behavioral Sciences (2 ed.). Hillsdale, NJ: Erlbaum. 
Cash, T. F. (2004). Body image: past, present, and future. Body Image, 1(1), 1-5. https://doi.org/10.1016/S17401445(03)00011-1

Cash, T. F. y Deagle, E. A. (1997). The nature and extent of body-image disturbances in anorexia nervosa and bulimia nervosa: a meta-analysis. The International Journal of Eating Disorders, 22(2), 107-126. https:// doi.org/10.1002/(SICI)1098-108X(199709)22:2\%3C107::AID-EAT1\%3E3.0.CO;2-J

Cash, T. F. y Smolak, L. (2011). Body Image: A Handbook of Science, Practice, and Prevention (2 ${ }^{\mathrm{a}}$ ed.). Nueva York, NY: Guilford Press.

Contreras, O. R., Gil-Madrona, P., Garcia-Lopez, L. M, Fernández-Bustos, J. G. y Pastor-Vicedo, J. C. (2012). Incidencia de un programa de Educación Física en la percepción de la propia imagen corporal. Revista de Educación, 357, 281-303. https://doi.org/10.4438/1988-592X-RE-2011-357-061

Craig, P., Halavatau, V., Comino, E. y Caterson, I. (1999). Perception of body side in the Tongan community: differences from and similarities to an Australian sample. International Journal of Obesity, 23(12), 12881294. https://doi.org/10.1038/sj.ijo.0801069.

Craig, P. L., Swinburn, B. A., Matenga-Smith, T., Matangi, H. y Vaughn, G. (1996). Do Polynesians still believe that big is beautiful? Comparison of body size perceptions and preferences of Cook Islands, Maori and Australians. The New Zealand medical journal, 109(1023), 200-203.

Cruzat-Mandich, C., Díaz-Castrillón, F., Lizana-Calderón, P., Aravena Winkler, M. y Haemmerli Delucchi, C. (2017a). Diferencias en imagen corporal de jóvenes con normopeso y con sobrepeso/obesidad. Nutrición Hospitalaria, 34(4), 847-855. http://dx.doi.org/10.20960/nh.824

Cruzat-Mandich, C., Díaz-Castrillón, F., Lizana-Calderón, P. y Castro, A. (2016). Comparación por sexo en imagen corporal, síntomas psicopatológicos y conductas alimentarias en jóvenes entre 14 y 25 años. Revista Médica de Chile, 144(6), 743-750. http://dx.doi.org/10.4067/S0034-98872016000600008

Cruzat-Mandich, C., Díaz-Castrillón, F., Pérez-Villalobos, C., Lizana, P., Moore, C., Simpson, S. y Oda-Montecinos, C. (2017b). Factor structure and reliability of the Multidimensional Body-Self Relations Questionnaire in Chilean youth. Eating and weight disorders, 24(2), 339-350. https://doi.org/10.1007/s40519-017-0411-z

Csendes, A. (2015). Número total de operaciones de patología digestiva alta en Chile año 2011. Revista Chilena de Cirugía, 67(1), 61-64. http://dx.doi.org/10.4067/S0718-40262015000100010

Díaz, M. C., Bilbao, G. M., Unikel, C., Muñoz, A., Escalante, E. I., y Parra, A. (2019). Relación entre estatus nutricional, insatisfacción corporal y conductas alimentarias de riesgo en estudiantes de nutrición. Revista mexicana de trastornos alimentarios, 10(1), 53-65. Recuperado de: https:/www.medigraphic.com/pdfs/ trastornos/mta-2019/mta191f.pdf

Durán, S., Rodríguez, M., Record, J., Barra, R., Olivares, R., Tapia, A., ... Neira, A. (2013). Autopercepción de la imagen corporal en estudiantes universitarios de Chile y Panamá. Revista Chilena de Nutrición, 40(1), 26-32. http://dx.doi.org/10.4067/S0717-75182013000100004

Durán, S., Valdés, P., Godoy, A. y Herrera, T. (2014). Hábitos alimentarios y condición física en estudiantes de pedagogía en educación física. Revista Chilena de Nutrición, 41(3), 251-259. http://dx.doi.org/10.4067/ S0717-75182014000300004

Fairburn, C. G., Cooper, Z. y Shafran, R. (2003). Cognitive behaviour therapy for eating disorders: A “transdiagnostic” theory and treatment. Behaviour Research and Therapy, 41(5), 509-528. https://doi.org/10.1016/ s0005-7967(02)00088-8

Organización de las Naciones Unidas para la Alimentación y la Agricultura. (2018, Octubre 15). Día Mundial de la Alimentación: Chile es el segundo país OCDE con la tasa más alta de obesidad. FAO. Recuperado de: http://www.fao.org/chile/noticias/detail-events/es/c/1157509/

Fehrman-Rosas, P., Delgado-Sánchez, C., Fuentes-Fuentes, J., Hidalgo-Fernández, A., Quintana-Muñoz, C., Yunge-Hidalgo, W., ... Durán-Agüero, S. (2016). Asociación entre autopercepción de imagen corporal y patrones alimentarios en estudiantes de Nutrición y Dietética. Nutrición Hospitalaria, 33(3), 649-654. http://dx.doi.org/10.20960/nh.274

Guzmán, S., Manrique, M., Raddatz, A., Nonero, E., Salinas, J., Achurra, P., ... Ibáñez, L. (2013). Experiencia de 18 años de cirugía de obesidad en la Pontificia Universidad Católica de Chile. Rev Med Chile, 141(5), 553-561. https://dx.doi.org/10.4067/S0034-98872013000500001

Gismero González, M. E. (2020). Factores de riesgo psicosociales en los Trastornos de la Conducta Alimentaria: Una revisión y algunas consideraciones para la prevención y la intervención [Psychosocial risk factors for Eating Disorders: a review and some considerations for prevention and intervention]. Revista de Psicoterapia, 31(115), 33-47. https://doi.org/10.33898/rdp.v31i115.354

Kirszman, D. y Salgueiro, M. C. (2015). Imagen Corporal. Dimensiones mediadoras en la psicopatología y modalidades de intervención [Body image: Mediating dimensions in psychopathology and types of intervention]. Revista Mexicana de Trastornos Alimentarios, 6(2), 129-136. http://dx.doi.org/10.1016/j.rmta.2015.10.004 
Ministerio de Salud.(MINSAL, 2017a). Encuesta Nacional de Salud 2016-2017. Primeros Resultados. Recuperado de: https://www.minsal.cl/wp-content/uploads/2017/11/ENS-2016-17_PRIMEROS-RESULTADOS.pdf

Ministerio de Salud (MINSAL, 2017b). Políticas públicas para combatir la obesidad en Chile: Ley 20.606 sobre la composición nutricional de los alimentos y su publicidad. Santiago de Chile, Chile: Ministerio de Salud, Gobierno de Chile.

Míguez, M., De la Montaña, J., González, J. y González, M. (2011). Concordancia entre la autopercepción de la imagen corporal y el estado nutricional en universitarios de Orense. Nutrición Hospitalaria, 26(3), 472479. https://doi.org/10.3305/nh.2011.26.3.4604

Mölbert, S. C., Klein, L., Thaler, A., Mohler, B. J., Brozzo, C., Martus, P. y Giel, K. E. (2017). Depictive and metric body size estimation in anorexia nervosa and bulimia nervosa: A systematic review and meta-analysis. Clinical Psychology Review, 57, 21-31. https://doi.org/10.1016/j.cpr.2017.08.005

Mujica, V., Leiva, E., Rojas, E., Díaz, N., Icaza, G. y Palomo, I. (2009). Discordancia en autopercepción de peso en población adulta de Talca. Revista Médica de Chile, 137, 76-82. http://dx.doi.org/10.4067/S003498872009000100011

Organización Mundial de la Salud (2017). 10 datos sobre la Obesidad. Recuperado de: https://www.who.int/ features/factfiles/obesity/es/

Pallan, M. J., Hiam, L. C., Duda, J. L. y Adab, P. (2011). Body image, body dissatisfaction and weight status in South Asian children: a cross-sectional study. BMC Public Health, 11, 21. https://doi.org/10.1186/14712458-11-21

Pruzinsky T. y Cash, T. (1990). Integrative themes in body-image development, deviance, and change. En T. F, Cash y T. Pruzinsky (Eds.), Body Images: Development, Deviance and Change (pp. 337-349). Nueva York, NY: The Guilford Press.

Raich, R. M. (2004). Una perspectiva desde la psicología de la salud de la imagen corporal. Avances en Psicología Latinoamericana, 22(1), 15-27. Recuperado de: https://revistas.urosario.edu.co/index.php/apl/article/ view/1261

Ramos, P., Rivera, F. y Moreno, C. (2010). Diferencias de sexo en imagen corporal, control de peso e índice de masa corporal de los adolescentes españoles. Psicothema, 22(1), 77-83. Recuperado de: http://www. psicothema.com/pdf/3699.pdf

Ramos, P., Rivera, F., Pérez, R. S., Lara, L. y Moreno, C. (2016). Diferencias de género en la imagen corporal y su importancia en el control de peso. Escritos de Psicología, 9(1), 42-50. https://dx.doi.org/10.5231/ psy.writ.2015.1409

Rodríguez, B., Oudhof, H., González-Arratia, N. I. y Unikel-Santocini, C. (2010). Desarrollo y validación de una escala para medir imagen corporal en mujeres jóvenes. Salud Mental, 33(4), 325-32.

Rudiger, J. A. y Winstead, B. A. (2013). Body talk and body-related co-rumination: associations with body image, eating attitudes, and psychological adjustment. Body Image, 10(4), 462-471. https://doi.org/10.1016/j. bodyim.2013.07.010

Slade, P. (1994). What is body image? Behaviour Research and Therapy, 32(5), 497-502. https://doi. org/10.1016/0005-7967(94)90136-8

Soto, M., Marín, B., Aguinaga, I., Guillén-Grima, F., Serrano, I., Canga, N., Hermoso de Mendoza. J., ... Annan, J. (2015). Análisis de la percepción de la imagen corporal que tienen los estudiantes universitarios de Navarra. Nutrición Hospitalaria, 31(5), 2269-2275. http://dx.doi.org/10.3305/nh.2015.31.5.7418

Streeter, V. M., Milhausen, R. R. y Buchholz, A. C. (2012). Body image, body mass index, and body composition in young adults. Canadian Journal of Dietetic Practice and Research, 73(2), 78-83. https://doi. org/10.3148/73.2.2012.78

Tiggemann, M., Martins, Y. y Churchett, L. (2008). Beyond muscles:unexplored parts for men’s body image. Journal of Health Psychology, 13(8), 1163-72. https://doi.org/10.1177/1359105308095971

Treasure, J. y Schmidt, U. (2013). The cognitive-interpersonal maintenance model of anorexia nervosa revisited: a summary of the evidence for cognitive, socio-emotional and interpersonal predisposing and perpetuating factors. Journal of Eating Disorders, 1, 13. https://doi.org/10.1186/2050-2974-1-13

Trejo, P. M., Castro, D., Facio, A., Mollinedo, F. E. y Valdez, G. (2010). Insatisfacción con la imagen corporal asociada al índice de masa corporal en adolescentes. Revista Cubana de Enfermería, 26(3), 144-54. Recuperado de: http://scielo.sld.cu/pdf/enf/v26n3/enf07310.pdf

Tremblay, L., Lovsin, T., Zecevic, C. y Larivière, M. (2011). Perceptions of self in 3-5-yearold children: A preliminary investigation into the early emergence of body dissatisfaction. Body Image, 8(3), 287-92. https:// doi.org/10.1016/j.bodyim.2011.04.004 
Van-der Hofstadt, C. J., Moncho, E., López, J. L., Abellán, C., Pérez, E., Tirado, S. y Rodríguez-Marín, J. (2012). Medida de la satisfacción del paciente e identificación de oportunidades de mejora en la preparación de pacientes de cirugía bariátrica. Revista de Calidad Asistencial, 27(5), 255-261. https://doi.org/10.1016/j. cali.2012.01.003

Ugarte, C., Quiñones, A. y Vicente, B. (2019). Predictores psicológicos de la re- ganancia del peso en pacientes bariátricos [Psychological predictors of the regain of weight in bariatric patients]. Terapia Psicológica, 37(3),199-209. http://dx.doi.org/10.4067/S0718-48082019000300199

Urzúa, A., Castro, S., Lillo, A. y Leal, C. (2009). Evaluación de los trastornos alimentarios: propiedades psicométricas del test EDI-2 en adolescentes escolarizados(as) de 13 a 18 años [Evaluation of Eating Disordres: Psychometric Properties of EDI-2 in Students 13 to 18 Years Old]. Revista Chilena de Nutrición, 36(4), 1063-1073. http://dx.doi.org/10.4067/S0717-75182009000400002

Wertheim, E. H. y Paxton, S. J. (2011). Body image development in adolescent girls. En T. F., Cash y L. Smolak (Eds), AHandbookofScience, Practice and Prevention (pp. 76-84). Londres, Reino Unido: The Gilford Press.

Williamson, D., Womble, L., Zucker, N., Reas, D., White M., Blouin, D. y Greenway, F. (2000). Body image assessment for obesity (BIA-O): development of a new procedure. International Journal of Obesity and Related Metabolic Disorders, 24(10), 1326-1332. https://doi.org/10.1038/sj.ijo.0801363 